ISSN 0103-8478

\title{
Redução na velocidade da nitrificação no solo após aplicação de cama de aviário com dicianodiamida
}

\author{
Reduction of the nitrification rate in the soil after addition of \\ poultry manure with dicyandiamide
}

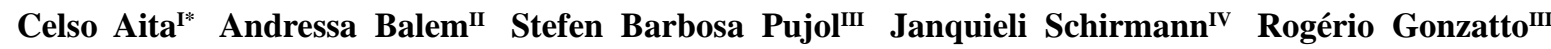 Diego Antonio Giacomini"II Patrícia Victória Vargas ${ }^{\mathrm{V}}$ Sandro José Giacomini ${ }^{\mathrm{I}}$}

\section{RESUMO}

A rápida nitrificação do nitrogênio $(N)$ amoniacal de fontes orgânicas e minerais no solo pode resultar em perdas de nitrato $\left(\mathrm{NO}_{3}^{-}\right)$para o ambiente. Uma estratégia para a redução dessas perdas envolve o uso de inibidores de nitrificação. $O$ objetivo do presente trabalho foi o de avaliar, em condições de laboratório, a eficiência da dicianodiamida (DCD), presente no produto Agrotain ${ }^{\circledR}$ Plus (AP), em inibir a nitrificação do $N$ amoniacal de cama de aviário (CA) no solo. Foram avaliados cinco tratamentos, sendo um com CA incorporada ao solo, três com CA incorporada ao solo com AP, nas doses de 3,5, 7,0 e

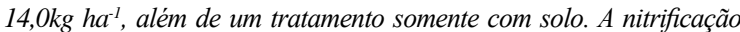
foi monitorada através da determinação periódica dos teores de $\mathrm{NH}_{4}^{+}$ e $\mathrm{NO}_{3}^{-}$no solo durante 69 dias. A maior taxa de nitrificação ocorren no tratamento em que a CA foi incorporada ao solo sem AP. As doses de 3,5 e 7,0kg de AP ha inibiram parcialmente a nitrificação do $\mathrm{N}$ amoniacal da CA na fase inicial da incubação, perdendo a eficiência em inibir esse processo no período entre 12 e 27 dias. $O$ tratamento com a maior dose de AP $\left(14 \mathrm{~kg} \mathrm{ha}^{-1}\right)$ foi aquele em que a DCD exerceu maior efeito inibitório da nitrificação, preservando maior quantidade de $\mathrm{NH}_{4}^{+}$e retardando o aparecimento de $\mathrm{NO}_{3}^{-}$no solo. Os resultados deste trabalho indicam que a DCD, contida no Agrotain ${ }^{\circledR}$ Plus, reduz a taxa de nitrificação do $N$ amoniacal da CA no solo, o que poderá contribuir à redução das perdas de $\mathrm{NO}_{3}^{-}$para o ambiente.

Palavras-chave: dinâmica do $\mathrm{N}$, lixiviação de $\mathrm{NO}_{3}^{-}$, desnitrificação, $D C D$

\section{ABSTRACT}

The fast nitrification of ammoniacal nitrogen $(N)$ of organic and mineral sources in soil can result in losses of nitrate $\left(\mathrm{NO}_{3}^{-}\right)$to the environment. One strategy to reduce these losses involves the use of nitrification inhibitors. The objective of this study was to evaluate, in laboratory conditions, the efficiency of Agrotain ${ }^{\circledR}$ Plus (AP), which contains dicyandiamide (DCD), as a nitrification inhibitor of ammoniacal nitrogen from poultry manure $(P M)$ in soil. Were evaluated five treatments, one with PM incorporated into the soil, three with PM incorporated into the soil with AP at the doses of 3.5, 7.0 e $14.0 \mathrm{~kg} \mathrm{ha}^{-1}$ and one treatment without PM and AP. Nitrification was monitored by measuring changes in soil mineral $\mathrm{N}\left(\mathrm{NH}_{4}^{+}\right.$and $\left.\mathrm{NO}_{3}^{-}\right)$periodically over 69 days. The highest rate of nitrification occurred in the treatment in which PM was incorporated into the soil without AP. The doses of 3.5 and $7.0 \mathrm{~kg} \mathrm{ha}^{-1}$ of AP inhibited partially the nitrification of ammoniacal nitrogen of PM in the initial phase of incubation, losing the ability to inhibit this process in the period between 12 and 27 days. The treatment with the higher dose of AP $\left(14 \mathrm{~kg} \mathrm{ha}^{-1}\right)$ was on in which the DCD exerted more inhibitory effect on nitrification, preserving most $\mathrm{NH}_{4}^{+}$and delaying the onset of $\mathrm{NO}_{3}$ - in soil. The results of this study indicate that the DCD, contained in the Agrotain ${ }^{\circledR}$ Plus, reduces the nitrification rate of ammoniacal nitrogen from $\mathrm{PM}$ in soil, that may contribute to reduce $\mathrm{NO}_{3}^{-}$losses to the environment.

Key words: $\mathrm{N}$ dynamics, $\mathrm{NO}_{3}$ - leaching, denitrification, $\mathrm{DCD}$

\section{INTRODUÇÃO}

O uso agrícola da cama de aviário, principalmente nas culturas do milho e feijão, é uma prática relativamente antiga nos estados do Sul do Brasil, onde a avicultura ocupa lugar de destaque na economia dessas regiões. Entre os nutrientes presentes na cama, o $\mathrm{N}$ é aquele que normalmente aparece em maiores concentrações, em função da dieta fornecida às aves.

'Departamento de Solos, Centro de Ciências Rurais (CCR), Universidade Federal de Santa Maria (UFSM), 97105-900, Santa Maria, RS,

Brasil. E-mail: celsoaita@gmail.com. *Autor para corespondência.

"Instituto Federal Faroupilha (IFF), Campus Alegrete, Alegrete, RS, Brasil.

IIIPrograma de Pós-graduação em Ciência do Solo, UFSM, Santa Maria, RS, Brasil.

Iv Programa de Pós-graduação em Ciência do Solo, Universidade Federal do Rio Grande do Sul (UFRGS), Porto Alegre, RS, Brasil.

${ }^{\mathrm{v}}$ Rota Agrícola, Passo Fundo, RS, Brasil. 
A rapidez com que o $\mathrm{N}$ amoniacal presente na cama de aviário é oxidado para $\mathrm{NO}_{3}^{-}$no solo irá determinar o potencial poluidor do ambiente, relativo a essa forma de $\mathrm{N}$ mineral. Isso por que o $\mathrm{NO}_{3}^{-}$poderá ser transferido, via escoamento superficial, aos mananciais de superfície, provocando a sua eutroficação e também ser lixiviado, contaminado a água do lençol freático (SINGH et al., 2008; ASING et al., 2008). Além disso, o $\mathrm{NO}_{3}$ - poderá ser usado, alternativamente ao oxigênio, na respiração de algumas bactérias, sendo reduzido até a forma gasosa de $\mathrm{N}_{2}$. Um dos gases intermediários desse processo microbiano de desnitrificação é o óxido nitroso $\left(\mathrm{N}_{2} \mathrm{O}\right)$, o qual é um potente gás de efeito estufa, além de contribuir na destruição da camada de ozônio (SUBBARAO et al., 2006). Portanto, a presença de $\mathrm{NO}_{3}{ }^{-}$no solo, em quantidades que excedam a demanda das plantas, aumenta o risco de poluição ambiental decorrente da fertilização nitrogenada, tanto de origem mineral quanto orgânica.

A adição de inibidores de nitrificação às fontes de $\mathrm{N}$ é uma das estratégias para retardar o aparecimento de $\mathrm{NO}_{3}^{-}$no solo e, com isso, melhorar o potencial fertilizante e mitigar a poluição ambiental por elas provocada (SUBBARAO et al., 2006). Um dos produtos mais utilizados nos últimos anos para esse fim é a DCD, que bloqueia a ação da enzima amônia monooxigenase, responsável pela oxidação de $\mathrm{NH}_{4}^{+}$para nitrito $\left(\mathrm{NO}_{2}^{-}\right)$em Nitrosomonas, na primeira etapa da nitrificação (SINGH et al., 2008). O uso de DCD, principalmente em locais onde ocorre o acúmulo de urina de vacas em lactação (DI et al., 2007) e em trabalhos com aplicação de dejetos de suínos ao solo (MKHABELA et al., 2006; DAMASCENO, 2010), tem retardado a nitrificação do $\mathrm{N}$ amoniacal dos dejetos, com redução na emissão de $\mathrm{N}_{2} \mathrm{O}$ e na lixiviação de $\mathrm{NO}_{3}^{-}$.

No Brasil, não foram encontrados trabalhos relatando o uso de DCD ou de outros produtos para inibir a nitrificação do $\mathrm{N}$ amoniacal de cama de aviário no solo. Mesmo em outros países, a aplicação de DCD para inibir a nitrificação em dejetos sólidos é um aspecto ainda relativamente pouco estudado (ASING et al., 2008). O elevado volume de cama de aviário produzido no Brasil, a rápida taxa de nitrificação do $\mathrm{N}$ contido na cama e as implicações ambientais negativas que isso pode representar evidenciam a necessidade de avaliar a eficiência de inibidores de nitrificação, quando aplicados ao solo juntamente com a cama de aviário. Esse foi o objetivo do presente trabalho, conduzido sob condições de laboratório, em que a cama de aviário foi incorporada ao solo com e sem a aplicação do produto Agrotain Plus, o qual contém $81 \%$ de DCD na sua composição.

\section{MATERIAL E MÉTODOS}

O trabalho consistiu na incubação em laboratório de um Argissolo Vermelho Distrófico arênico, coletado na camada de $0-10 \mathrm{~cm}$ e com as seguintes características químicas e físicas: $\mathrm{pH}$ água $(1: 1)=5,0 ; \mathrm{MO}=26 \mathrm{~g} \mathrm{~kg}^{-1} ;$ Argila $=24 \mathrm{~g} \mathrm{~kg}^{-1} ; \mathrm{P}(\mathrm{mg}$ $\left.\mathrm{dm}^{-3}\right)=3,0 ; \mathrm{K}\left(\mathrm{mg} \mathrm{dm}^{-3}\right)=48 ; \mathrm{Al}\left(\mathrm{cmol}_{\mathrm{c}} \mathrm{dm}^{-3}\right)=2,0 ; \mathrm{Ca}$ $\left(\mathrm{cmol}_{\mathrm{c}} \mathrm{dm}^{-3}\right)=1,4 ; \mathrm{Mg}\left(\mathrm{cmol}_{\mathrm{c}} \mathrm{dm}^{-3}\right)=0,6$.

$\mathrm{Na} \mathrm{CA}$, em que foram criados 10 lotes de frangos de corte, foram determinados, sem secagem prévia e utilizando a metodologia descrita em TEDESCO et al. (1995), o pH, a matéria seca e os teores de $\mathrm{N}$ total Kjeldahl (NTK) e $\mathrm{N}$ mineral $\left(\mathrm{NH}_{4}^{+}{ }^{+} \mathrm{NO}_{2}{ }^{-}+\mathrm{NO}_{3}{ }^{-}\right)$. O teor de carbono (C) da $\mathrm{CA}$ foi determinado em analisador elementar CHNS (modelo FlashEA 1112, marca Thermo Electron), nas amostras secas a $65^{\circ} \mathrm{C}$. As principais características da CA, bem como as quantidades adicionadas ao solo de matéria seca, carbono e nitrogênio encontram-se na tabela 1 .

O produto avaliado quanto a sua eficiência em inibir a nitrificação do $\mathrm{N}$ amoniacal da $\mathrm{CA}$ foi o Agrotain ${ }^{\circledR}$ Plus (AP). O AP possui na sua formulação $81,0 \%$ de DCD, a qual atua especificamente sobre a inibição da enzima amônia monooxigenase, responsável pela primeira etapa da nitrificação (SUBBARAO et al., 2006).

O delineamento experimental utilizado foi o inteiramente casualizado, com 4 repetições dos seguintes tratamentos: T1 - Solo (S) (testemunha); T2$\mathrm{S}+\mathrm{CA} ; \mathrm{T} 3-\mathrm{S}+\mathrm{CA}+3,5 \mathrm{~kg} \mathrm{ha}^{-1} \mathrm{AP} ; \mathrm{T} 4-\mathrm{S}+\mathrm{CA}+7,0 \mathrm{~kg}$ ha $^{-1}$ AP; T5- S + CA + 14,0kg ha-1 AP. A dose equivalente de CA aplicada foi de $4,7 \mathrm{Mg} \mathrm{ha}^{-1}$ (base seca) e as quantidades de $\mathrm{C}$ e $\mathrm{N}$ total adicionadas ao solo (em mg kg-1 de solo seco) com o AP foram de, respectivamente, 1,57 e $3,38\left(3,5 \mathrm{~kg} \mathrm{ha}^{-1} \mathrm{de} \mathrm{AP}\right), 3,14$ e $6,76\left(7,0 \mathrm{~kg} \mathrm{ha}^{-1}\right.$ de AP) e de 6,28 e $13,52(14,0 \mathrm{~kg}$ $\mathrm{ha}^{-1}$ de AP).

Os tratamentos foram aplicados ao solo em recipientes de acrílico, com 5,0 cm de altura e $5,1 \mathrm{~cm}$ de diâmetro, com capacidade de 102,1mL. Para avaliação do $\mathrm{N}$ mineral do solo, os recipientes de acrílico foram acondicionados em potes de vidro com capacidade de $2 \mathrm{~L}$, com quatro repetições por tratamento. Para evitar a deficiência de $\mathrm{O}_{2}$, o que limitaria a decomposição aeróbica dos materiais orgânicos, os frascos foram abertos semanalmente durante 15 minutos para aeração.

A quantidade de solo colocada em cada recipiente de acrílico foi de 142,1g com 13,8\% de umidade ( $80 \%$ da capacidade de campo). O produto AP foi adicionado ao solo, juntamente com 
Tabela 1 - Teores de matéria seca (MS), carbono (C) e nitrogênio $(\mathrm{N})$ na cama de aviário, valores da relação $\mathrm{C} / \mathrm{N}$ e do pH e quantidades de MS, $\mathrm{C}$ e $\mathrm{N}$ adicionadas a um Argissolo Vermelho Distrófico arênico com a cama de aviário.

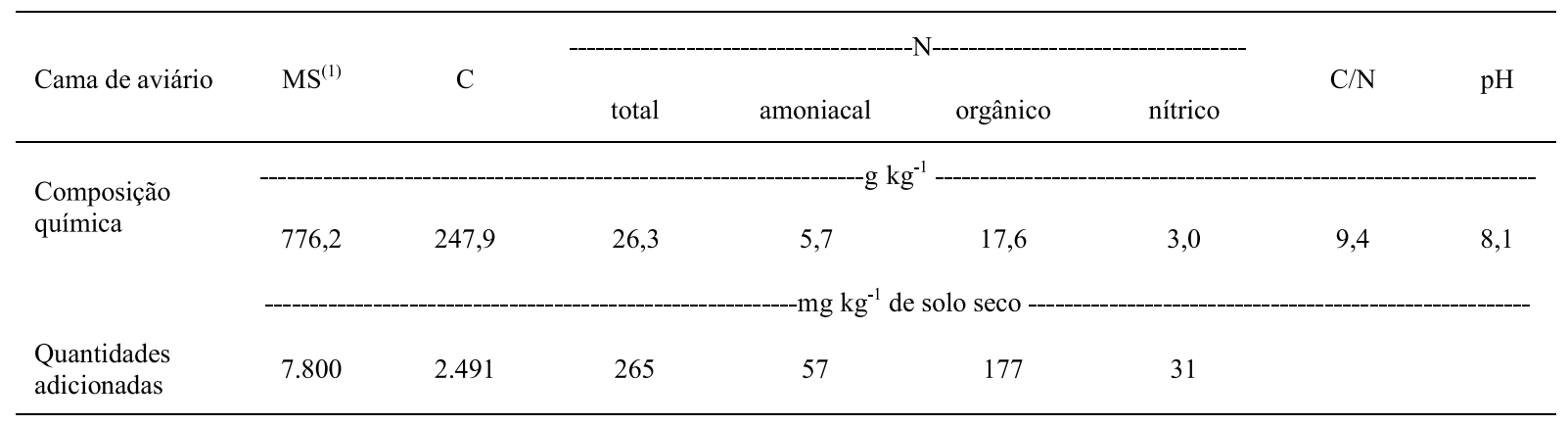

${ }^{(1)}$ Os valores de MS, C e N da cama de aviário referem-se a base úmida.

a CA. Para a mistura do AP na CA, a quantidade de produto equivalente a cada uma das doses foi inicialmente dissolvida em $50 \mathrm{~mL}$ de água destilada e essa solução foi misturada a $100 \mathrm{~g}$ de CA. Após, a mistura AP + CA foi uniformemente incorporada ao solo de cada tratamento, com auxílio de espátula, sendo adicionada nos recipientes de acrílico em duas etapas. $\mathrm{Na}$ primeira, foram adicionados $71,05 \mathrm{~g}$ de solo, compactando-o até a altura de $2,5 \mathrm{~cm}$ no frasco de acrílico. Na segunda, adicionou-se o restante do solo (71,05g), compactando-o até a altura de $5 \mathrm{~cm}$. Dessa forma, o solo do frasco atingiu uma densidade final de aproximadamente $1,2 \mathrm{~g} \mathrm{~cm}^{-3}$.

Todas as unidades experimentais foram mantidas em uma incubadora a $25^{\circ} \mathrm{C}$, na ausência de luminosidade, por um período de 69 dias. A umidade do solo foi mantida durante a incubação através da pesagem das amostras e, quando necessário, foi adicionada água destilada na superfície de cada frasco.

A nitrificação foi avaliada através da determinação periódica dos teores de $\mathrm{N}$ mineral do solo $\left(\mathrm{NH}_{4}^{+} \mathrm{e} \mathrm{NO}_{2}^{-}+\mathrm{NO}_{3}^{-}\right)$, iniciando-se duas (02) horas após a aplicação dos tratamentos (tempo 0) e repetindo-se a análise aos 3, 6, 12, 27, 54 e 69 dias após o início da incubação. Em cada avaliação, o $\mathrm{N}$ mineral foi extraído de $20 \mathrm{~g}$ do solo com uma solução de $80 \mathrm{~mL}$ de $\mathrm{KCl} 1 \mathrm{~mol}$ $\mathrm{L}^{-1}$ e determinado por destilação, conforme TEDESCO et al. (1995).

O efeito dos tratamentos sobre as variáveis em estudo foi avaliado através da análise de variância e as médias foram comparadas entre si pelo teste de Tukey a 5\% de probabilidade, sendo, para tanto, usado o programa STATISTICA 5.1.

\section{RESULTADOS E DISCUSSÃO}

Analisando conjuntamente os resultados relativos ao $\mathrm{N}$ na forma amoniacal e nítrica no solo dos quatro tratamentos com adição de CA (Figura 1), observa-se que a redução nas quantidades de $\mathrm{NH}_{4}^{+}$ (Figura 1A), especialmente na primeira semana de incubação, foi mais acentuada do que o aumento das quantidades de $\mathrm{NO}_{3}^{-}$(Figura 1b). Isso evidencia que a nitrificação não foi o único processo responsável pelo desparecimento do $\mathrm{N}$ amoniacal, tanto daquele adicionado ao solo com a CA como daquele produzido pela amonificação do $\mathrm{N}$ orgânico nessa fase inicial de decomposição da cama no solo. Os outros destinos prováveis para o $\mathrm{N}$ amoniacal poderiam ter sido a volatilização de $\mathrm{NH}_{3}$ e a sua imobilização temporária pela população microbiana. Todavia, a contribuição da volatilização de amônia nessa rápida diminuição inicial do $\mathrm{NH}_{4}^{+}$do solo deve ter sido pequena, em razão das condições de incubação, pouco favoráveis à ocorrência desse processo físico-químico de perda de N. Na incubação, a cama foi incorporada ao solo, o que protege o $\mathrm{N}$ amoniacal da volatilização (SOMMER et al., 2003). Além disso, os frascos fechados impediram a ação dos ventos, um dos fatores que pode favorecer a emissão de $\mathrm{NH}_{3}$ para a atmosfera (SOMMER et al., 2003). Portanto, além da nitrificação, a imobilização microbiana deve ter sido a outra causa determinante da rápida redução ocorrida nos teores de $\mathrm{NH}_{4}{ }^{+}$dos tratamentos com CA, no período inicial de incubação.

Apesar de a CA apresentar, normalmente, baixa relação $\mathrm{C} / \mathrm{N}$ (Tabela 1 ), a imobilização de $\mathrm{N}$ tem sido observada em outros trabalhos, como, por exemplo, na incubação conduzida durante 120 dias por AZEEZ \& AVERBEKE (2010) a uma temperatura de $23^{\circ} \mathrm{C}$ e com uma $\mathrm{CA}$ com relação $\mathrm{C} / \mathrm{N}$ de 9,6/1. Essa imobilização de $\mathrm{N}$ pode ser atribuída ao fornecimento de carbono e energia à população microbiana heterotrófica através da CA, o que estimula sua atividade e, por consequência, a demanda em N para o metabolismo celular. Quando a assimilação microbiana do $\mathrm{N}$ disponível do solo é 
maior do que a liberação desse nutriente através do processo de mineralização, ocorre a imobilização líquida de $\mathrm{N}$, detectada através da diminuição dos teores de $\mathrm{N}$ mineral do solo (AZEEZ \& AVERBEKE, 2010). A ocorrência desse processo de imobilização líquida de $\mathrm{N}$ durante a decomposição da CA no solo, bem como a sua magnitude, deve estar relacionada, principalmente, ao tipo de material orgânico empregado na cama, sobre a qual serão criadas as aves.

Os resultados da figura 1a mostram que, no tratamento com $\mathrm{CA}$ sem $\mathrm{AP}$, a diminuição líquida da quantidade de $\mathrm{N}^{-} \mathrm{NH}_{4}{ }^{+}$aos 54 dias foi de aproximadamente $50 \mathrm{mg}$ de $\mathrm{N}_{-} \mathrm{NH}_{4}^{+} \mathrm{kg}^{-1}$ de solo. Todavia, no mesmo período, o acúmulo líquido de $\mathrm{NO}_{3}^{-}$proveniente da cama foi próximo a $100 \mathrm{mg}$ $\mathrm{kg}^{-1}$ de solo (Figura 2). A não correspondência entre a diminuição do $\mathrm{N}$ amoniacal e o aumento do $\mathrm{N}$ nítrico também ocorreu nos trabalhos de SISTANI et al. (2008) e AZEEZ \& AVERBEKE (2010), evidenciando que, no caso da $\mathrm{CA}$, o $\mathrm{N}$ orgânico é rapidamente mineralizado pelos microrganismos do solo e o $\mathrm{N}$ amoniacal resultante desse processo é rapidamente nitrificado no solo. Por isso, em CA, os inibidores deverão atuar tanto na inibição da nitrificação do $\mathrm{N}$ amoniacal já presente na cama, no momento da sua aplicação ao solo, como no $\mathrm{N}$ proveniente da mineralização do $\mathrm{N}$ orgânico pelos microrganismos do solo.

O efeito do inibidor de nitrificação é evidenciado na figura 1, através da comparação dos teores de $\mathrm{NH}_{4}^{+}$entre os quatro tratamentos com aplicação de CA. Nos primeiros três dias, não houve diferença entre esses quatro tratamentos, em que houve

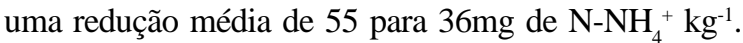
Somente após esse período, a variação temporal do $\mathrm{NH}_{4}^{+}$no solo ocorreu de modo distinto. Enquanto no tratamento com $\mathrm{CA}$ e sem $\mathrm{AP} \mathrm{o} \mathrm{NH}_{4}^{+}$do solo diminuiu continuamente até os 54 dias, nos tratamentos com CA e AP, nas doses de 3,5 e 7,0kg ha' ${ }^{-1} \mathrm{o} \mathrm{NH}_{4}^{+}$se manteve relativamente constante até 12 e 27 dias, respectivamente. Já na maior dose de AP $\left(14 \mathrm{~kg} \mathrm{ha}^{-1}\right)$, o teor de $\mathrm{NH}_{4}^{+}$aumentou do terceiro ao vigésimo sétimo dia e se manteve até o final do experimento entre 50,0 e $60,0 \mathrm{mg}$ de $\mathrm{N}-\mathrm{NH}_{4}^{+} \mathrm{kg}^{-1}$. Essa maior proporção de $\mathrm{NH}_{4}^{+}$no solo com o aumento da dose de AP revela o efeito inibitório da dicianodiamida (DCD), tanto sobre $\mathrm{o} \mathrm{NH}_{4}^{+}$presente inicialmente na CA como também do $\mathrm{NH}_{4}^{+}$produzido pelos microrganismos heterotróficos responsáveis pela sua decomposição no solo.

A avaliação conjunta da variação dos teores de $\mathrm{NH}_{4}^{+}$e $\mathrm{NO}_{3}^{-}$no solo de cada tratamento (Figura 1) fornece uma informação mais precisa do efeito

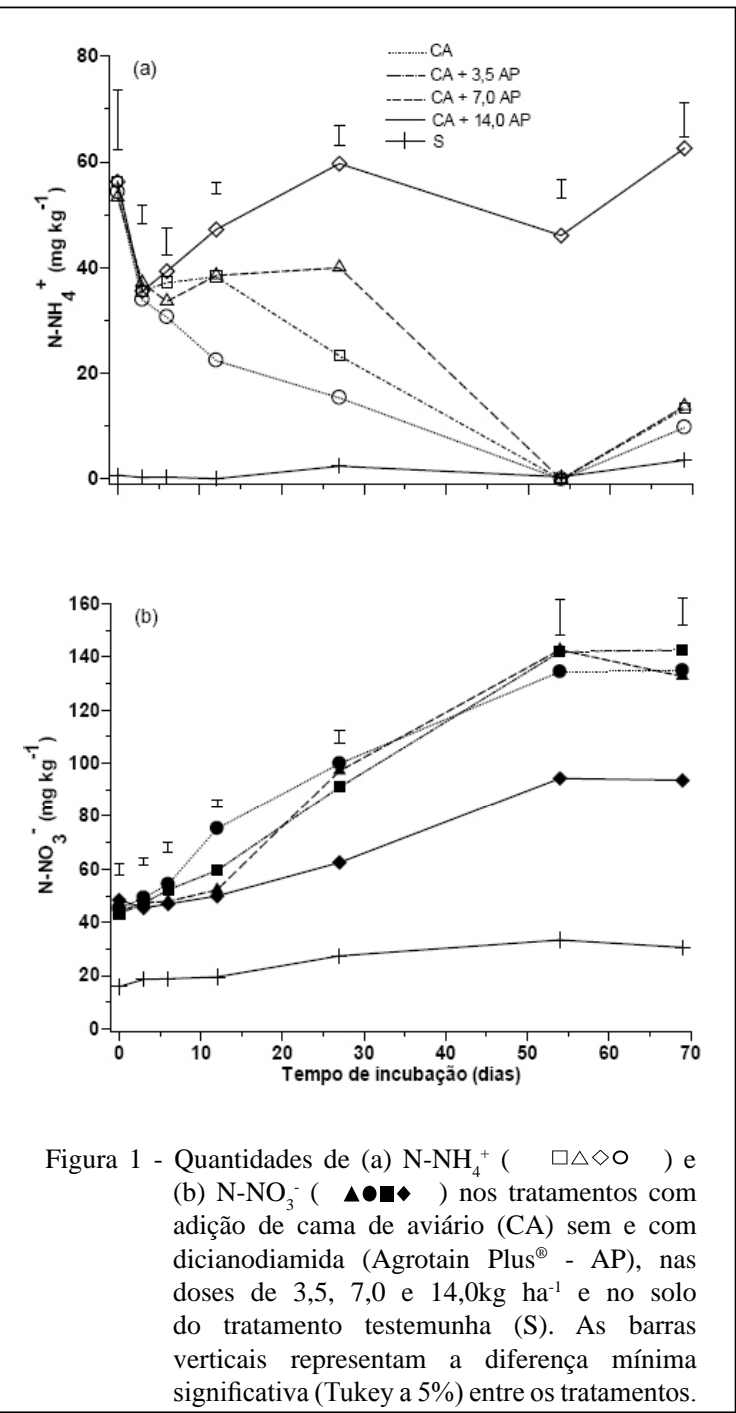

inibitório do AP sobre a nitrificação. Observa-se que, nos tratamentos em que a cama foi adicionada ao solo juntamente com as doses de 3,5 e 7,0 $\mathrm{kg} \mathrm{ha}^{-1}$ de AP, o teor de $\mathrm{NO}_{3}{ }^{-}$no solo desses tratamentos não diferiu do tratamento em que a CA não recebeu AP (Figura 1b), já a partir de 27 dias. O tratamento que apresentou os menores teores de $\mathrm{N}^{-\mathrm{NO}_{3}}{ }^{-}$no solo em praticamente todo o período de incubação foi aquele em que a cama foi incorporada ao solo com a maior dose de AP (14kg ha-1). Mesmo aos 69 dias após a adição da cama ao solo, o teor de $\mathrm{N}_{-} \mathrm{NO}_{3}$ - desse tratamento foi inferior à média dos outros três tratamentos com cama em $43 \mathrm{mg} \mathrm{kg}^{-1}$. Esses resultados indicam que a dicianodiamida (DCD), contida no produto AP, inibiu a nitrificação do $\mathrm{N}$ amoniacal da $\mathrm{CA}$, aumentando o tempo de permanência dessa forma de $\mathrm{N}$ mineral no solo. Todavia, esse efeito foi detectado de modo mais 


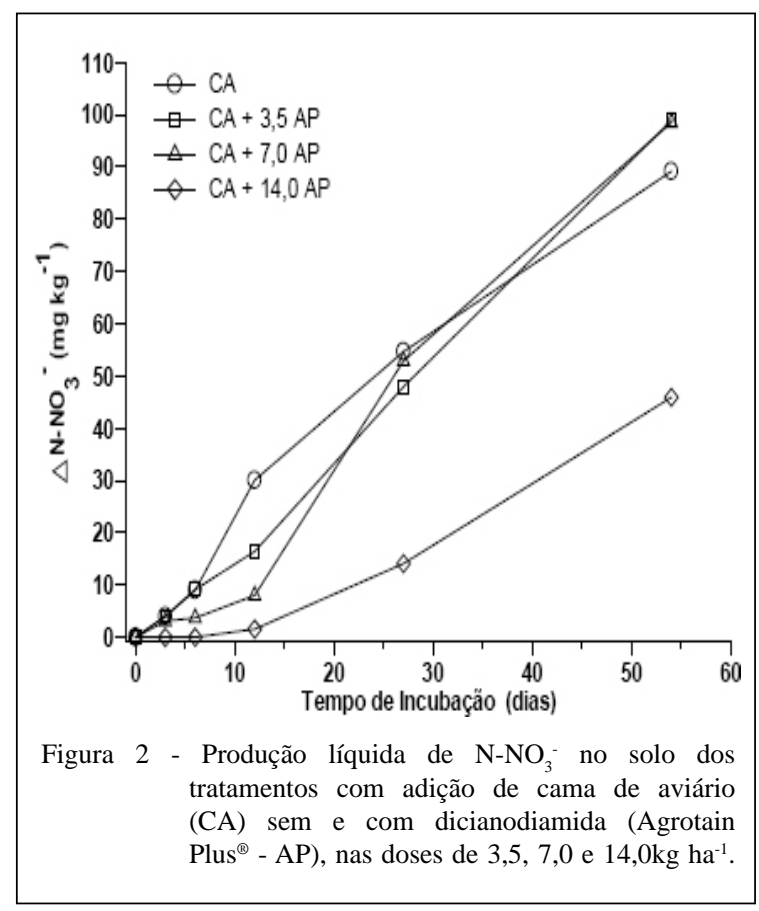

significativo e prolongado apenas na dose de $14 \mathrm{~kg}$ $\mathrm{ha}^{-1}$ de AP (11,34kg ha-1 de DCD).

Não foram encontrados resultados publicados envolvendo o uso de DCD para inibir a nitrificação em CA, para compará-los aos resultados do presente trabalho. O único trabalho encontrado, em que esse inibidor foi aplicado a dejetos sólidos, é aquele realizado por ASING et al. (2008) na Nova Zelândia, em que a dose de $13 \mathrm{~kg}$ de DCD ha ${ }^{-1}$ foi aplicada num fertilizante orgânico peletizado, produzido a partir de esterco de ovelha e denominado de Garden galore. Esses autores encontraram que a aplicação de DCD manteve maiores concentrações de $\mathrm{N}$ mineral na forma de $\mathrm{NH}_{4}{ }^{+}$no solo, além de reduzir a emissão de $\mathrm{N}_{2} \mathrm{O}$ e a lixiviação de $\mathrm{NO}_{3}^{-}$.

A partir dos resultados relativos ao acúmulo de $\mathrm{NO}_{3}^{-}$(Figura 1B), foi possível calcular o acúmulo de $\mathrm{NO}_{3}^{-}$no solo, proveniente do $\mathrm{N}$ da CA. Para isso, o teor de $\mathrm{NO}_{3}^{-}$encontrado no solo do tratamento testemunha (S) foi descontado dos quatro tratamentos com CA. É importante destacar que esse cálculo permite inferir apenas sobre a nitrificação líquida ocorrida e não sobre a nitrificação total ou bruta. Isso porque, da quantidade total de $\mathrm{NO}_{3}^{-}$produzida pela oxidação microbiana do $\mathrm{NH}_{4}^{+}$, uma parte poderá ser removida do solo através dos processos microbianos de desnitrificação e imobilização pela população microbiana heterotrófica. É pouco provável que a desnitrificação tenha removido quantidades significativas de $\mathrm{NO}_{3}^{-}$do sistema, uma vez que a condição aeróbica empregada na incubação é inibitória à ação das bactérias anaeróbias facultativas responsáveis por esse processo.

Comparando o efeito inibitório da nitrificação entre as três doses de AP, aplicadas ao solo juntamente com a CA, observa-se, através dos resultados da figura 2, que o acúmulo líquido de $\mathrm{NO}_{3}^{-}$ no solo do tratamento com a dose de $14 \mathrm{~kg} \mathrm{ha}^{-1} \mathrm{AP}$ só iniciou após 12 dias de incubação. Já nas doses de 3,5 e 7,0 $\mathrm{kg} \mathrm{ha}^{-1}$ de $\mathrm{AP}$, constatou-se acúmulo de $\mathrm{NO}_{3}^{-}$no solo desde o início do experimento, sendo que, aos 27

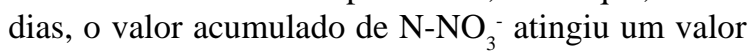
médio de $50 \mathrm{mg} \mathrm{kg}^{-1}$, aproximando-se do tratamento com uso de CA sem AP e superando o tratamento com $14 \mathrm{~kg}$ de AP ha-1 em $35 \mathrm{mg} \mathrm{kg}^{-1}$. Tais resultados evidenciam que o AP aplicado na dose de $14 \mathrm{~kg} \mathrm{ha}^{-1}$ inibiu completamente a nitrificação do $\mathrm{N}$ amoniacal da CA nos primeiros 12 dias e parcialmente após esse período. Mesmo quando iniciou a nitrificação nesse tratamento, aos 12 dias, o acúmulo de $\mathrm{NO}_{3}^{-}$, no período de 12 a 54 dias, ocorreu a uma taxa de $1,07 \mathrm{mg} \mathrm{kg}^{-1}$ de solo dia ${ }^{-1}$, que foi aproximadamente duas vezes menor do que a média dos tratamentos com 3,5 e 7,0 kg ha- ${ }^{-1}$ AP $\left(2,02 \mathrm{mg} \mathrm{kg}^{-1}\right.$ de solo dia $\left.{ }^{-1}\right)$ e $23 \%$ inferior ao tratamento com cama sem AP $\left(1,39 \mathrm{mg} \mathrm{kg}^{-1}\right.$ de solo dia $\left.{ }^{-1}\right)$. Nas doses de 3,5 e 7,0 kg ha $^{-1} \mathrm{o}$ AP perdeu a capacidade em inibir a nitrificação do $\mathrm{N}$ amoniacal da cama, no período entre 12 e 27 dias após a sua aplicação. O fato de a DCD, que é a substância inibidora da nitrificação, ser biodegradável no solo pode explicar esses resultados.

Os resultados deste trabalho evidenciam que, para um efeito prolongado da inibição da nitrificação do $\mathrm{N}$ amoniacal de $\mathrm{CA}$, o produto $\mathrm{AP}$, contendo $81 \%$ do inibidor DCD, deverá ser aplicado ao solo, juntamente com a cama, em doses superiores a $7 \mathrm{~kg} \mathrm{ha}^{-1}$. Ao realizar uma revisão crítica sobre desafios e oportunidades envolvendo estratégias para controlar a nitrificação em sistemas agrícolas, SUBBARAO et al. (2006) destacam que a DCD é solúvel em água e biodegradável no solo. Nessa mesma revisão, os autores enfatizam que a quantidade necessária de DCD para inibir a nitrificação varia de 10 a $50 \mathrm{mg} \mathrm{kg}^{-1}$ de solo e que esse efeito inibitório dura de 4 a 8 semanas. Na presente incubação, em que não houve saída de DCD do sistema, pelo fato de estar isolado da ação das chuvas, a inibição da nitrificação somente foi efetiva na dose de $14 \mathrm{~kg} \mathrm{ha}^{-1}$ de AP (18,8 $\mathrm{mg}$ de DCD kg-1 de solo).

Embora a dicianodiamida tenha retardado a nitrificação do $\mathrm{N}$ amoniacal da $\mathrm{CA}$ no presente trabalho, é importante confirmar esses resultados em experimentos de campo, sob condições variáveis de clima e de solo. Os resultados obtidos sugerem 
que as doses de AP a serem testadas no campo sejam superiores a $7,0 \mathrm{~kg} \mathrm{ha}^{-1}$. Nesses trabalhos, é importante avaliar também o efeito do produto sobre outros processos do ciclo do $\mathrm{N}$, com destaque para as perdas de $\mathrm{N}$ por volatilização de $\mathrm{NH}_{3}$, lixiviação de $\mathrm{NO}_{3}{ }^{-}$e desnitrificação.

\section{CONCLUSÃO}

A DCD, contida no produto Agrotain ${ }^{\circledR}$ Plus, apresentou efeito inibidor sobre a nitrificação do $\mathrm{N}$ amoniacal da cama de aviário no solo, sendo que, nas doses de 3,5 e 7,0kg de Agrotain $^{\circledR}$ Plus ha $^{-1}$, a inibição ocorreu apenas nos primeiros 12 dias, enquanto, na dose de $14 \mathrm{~kg} \mathrm{ha}^{-1}(11,34 \mathrm{~kg}$ de DCD ha- $)$, esse efeito ocorreu até o final do experimento, aos 69 dias.

\section{REFERÊNCIAS}

ASING, J. et al. Assessment of nitrogen losses from urea and an organic manure with and without nitrification inhibitor, dicyandiamide, applied to lettuce under glasshouse conditions. Australin Journal of Soil Research, v.46, p.535-541, 2008 Disponível em: <http://www.publish.csiro.au/paper/SR07206. htm>. Acesso em: 20 jul. 2011. doi: 10.1071/SR07206.

AZEEZ, J.O.; VAN AVERBEKE, W. Nitrogen mineralization potential of three animal manures applied on a sandy clay loam soil. Bioresource Technology, v.101, p.5645-5651, 2010. Disponível em: <http://www.sciencedirect.com/science/article/pii/ S0960852410002051>. Acesso em: 23 jul. 2011. doi: 10.1016/j. biortech.2010.01.119.

DAMASCENO, F. Injeção de dejetos de suínos no solo e inibidor de nitrificação como estratégias para reduzir as emissões de amônia e óxido nitroso. 2010. 121f. Dissertação (Mestrado em Ciência do Solo) - Curso de Pós-graduação em Ciência do Solo, Universidade Federal de Santa Maria, RS.
DI, H.J. et al. Comparison of the effectiveness of a nitrification inhibitor, dicyandiamide, in reducing nitrous oxide emissions in four different soils under different climatic and management conditions. Soil Use and Management, v.23, p.1-9, 2007. Disponível em: <http://onlinelibrary.wiley.com/doi/10.1111/ j.1475-2743.2006.00057.x/pdf>. Acesso em: 25 jul. 2011. doi: 10.1111/j.1475-2743.2006.00057.x.

MKHABELA, M.S. et al. Ammonia and nitrous oxide emissions from two acidic soils of Nova Scotia fertilized with liquid hog manure mixed with or without dicyandiamide. Chemosphere, v.65, p.1381-1387, 2006. Disponível em: <http://www.sciencedirect. com/science/article/pii/S0045653506004590>. Acesso em: 22 jul. 2011. doi: 10.1016/j.chemosphere.2006.04.062.

SINGH, J. et al. The role of inhibitors in the bioavailability and mitigation of nitrogen losses in grassland ecosystems. Developments in Soil Science, v.32, p.329-362, 2008. Disponível em: <http:// www.sciencedirect.com/science/article/pii/S0166248107320151>. Acesso em: 22 jul. 2011. doi:10.1016/S0166-2481(07)32015-1.

SISTANI, K.R. et al. Laboratory and field evaluation of broiler litter nitrogen mineralization. Bioresource Technology, v.99, p.26032611, 2008. Disponível em: <http://www.sciencedirect.com/ science/article/pii/S0960852407004038>. Acesso em: 24 jul. 2011. doi: 10.1016/j.biortech.2007.04.069.

SOMMER, S.G. et al. Processes controlling ammonia emissions from livestock slurry in the field. European Journal of Agronomy, v.19, p.465-486, 2003. Disponível em: < http://www.sciencedirect. com/science/article/pii/S1161030103000376>. Acesso em: 30 ago. 2011. doi: 10.1016/S1161-0301(03)00037-6.

SUBBARAO, G.V. et al. Scope and strategies for regulation of nitrification in agricultural systems - challenges and opportunities. Critical Reviews in Plant Sciences, v.25, p.303-335, 2006. Disponível em: <http://www.tandf.co.uk/journals/titles/07352689. asp>. Acesso em: 30 ago. 2011. doi: 10.1080/07352680600794232.

TEDESCO, M.J. et al. Análises de solo, plantas e outros materiais. Porto Alegre: Universidade Federal do Rio Grande do Sul, Faculdade de Agronomia, 1995. 174p. (Boletim Técnico de Solos, 5). 